\title{
Aryltetralols from Holostylis reniformis and syntheses of lignan analogous
}

\author{
Marcos D.P. Pereira ${ }^{\mathrm{a}}$, Matheus R. Ferreira ${ }^{\mathrm{a}}$, Gisele B. Messiano ${ }^{\mathrm{a}, 1}$, Isabela Penna Cerávolo ${ }^{\mathrm{b}}$ \\ , Lucia M.X. Lopes ${ }^{\mathrm{a}, *}$, Antoniana U. Krettli ${ }^{\mathrm{b}, * *}$ \\ a Institute of Chemistry, São Paulo State University, UNESP, C.P. 355, 14801-970, Araraquara, SP, Brazil

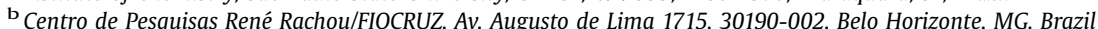

\section{A R T I C L E I N F O}

\section{Article history:}

Received 18 March 2015

Received in revised form 26 May 2015

Accepted 4 June 2015

Available online 25 June 2015

\section{Keywords:}

Holostylis reniformis

Aristolochiaceae

Lignans

Aryltetralol

Arytetralene

Antiplasmodial activity

\begin{abstract}
A B S T R A C T
Two new lignans, an aryltetralol and its methyl ether analogous, were isolated from Holostylis reniformis (Aristolochiaceae) together with futokadsurin $\mathrm{C}$ and $(-)-8^{\prime}-$ epi-aristoligone. The latter was also obtained as an enantiomeric mixture by synthesis and was transformed into aryltetralols and aryltetralenes that were subjected to chiral-HPLC separations. The compound structures were determined by spectroscopic methods. Several of these lignans had their antiplasmodial activity (against Plasmodium falciparum, W2 clone, anti-HRPII) and toxicity to mammalian kidney cells $\left(\mathrm{MDL}_{50}\right)$ evaluated. (-)-Cyclogalgravin and (-)-aristoligol exhibited activity ( $\mathrm{IC}_{50} \sim 10.8$ and $8.4 \mu \mathrm{M}$, respectively), the latter exhibited lower toxicity. (c) 2015 Phytochemical Society of Europe. Published by Elsevier B.V. All rights reserved.
\end{abstract}

\section{Introduction}

Holostylis reniformis Duch. (Aristolochiaceae) synthesizes a variety of seco compounds, including lignans and sesquiterpenes (da Silva and Lopes, 2004, 2006; Lopes et al., 2012; Martins et al., 2014; Pereira et al., 2012). More than 25 aryltetralone and furan lignans without oxygenation at $C-9,9^{\prime}$ have been isolated from extracts of this species (da Silva and Lopes, 2004, 2006; Lopes et al., 2012), and isoeugenol is shown to be their biosynthetic intermediate (da Silva and Lopes, 2004, 2006; Messiano et al., 2008, 2009). The anti-Plasmodium falciparum activities and toxicities of lignans and extracts obtained from $\mathrm{H}$. reniformis have been demonstrated (da Silva et al., 2004; da Silva and Lopes, 2004; de Andrade-Neto et al., 2007). As part of our continuing studies on the chemical constituents of $H$. reniformis, in this paper we report the results of the in vitro antiplasmodial and toxicity evaluation of two new natural lignans ( $\mathbf{1}$ and $\mathbf{2}$ ) and of aryltetralone, aryltetralol,

\footnotetext{
* Corresponding author. Fax: +55 1633019692.

** Corresponding author. Fax: +55 3132953115 .

E-mail addresses: mpelicon@bol.com.br (M.D.P. Pereira)

matheusrf_sud@hotmail.com (M.R. Ferreira),gbaraldi@ifsp.edu.br(G.B. Messiano) , ceravolo@cpqrr.fiocruz.br (I.P. Cerávolo), lopesxl@iq.unesp.br (L.M.X. Lopes), akrettli@cpqrr.fiocruz.br (A.U. Krettli).

1 Present address: Instituto Federal de São Paulo, R. Stéfano D’Avassi 625, 15991 502 Matão, SP, Brazil.
}

and aryltetralene lignans obtained by regioselective syntheses. These lignans belong to $2,7^{\prime}$-cyclolignan subclass of lignans or arylnaphthalene type of lignans. Lignans of this subclass, without oxygenation at C-9, $9^{\prime}$, have already been obtained by syntheses using commercially available compounds (Kuo and Lin, 1993; Takeya et al., 1983; Yvon et al., 2001), via transformations of 7,7'-epoxylignans (furan lignans) (Blears and Haworth, 1958; Crossley and Djerassi, 1962; Purushothaman et al., 1984), and by biotransformations from fungi (Messiano et al., 2010). However, stereoselective syntheses of 2,7'-cyclolignans normally involve more than 10 steps (Peng et al., 2013; Rye and Barker, 2011), and those regioselective carried out in fewer steps showed poor yields (Barba et al., 1990; Iguchi et al., 1978). Aiming to obtain lignan analogous to advances in pharmacological studies of these lignans, this report describes a sequence for the syntheses of aryltetralone, aryltetralol, and aryltetralene lignans in four, five, and six steps, successively by improving a route for obtaining aryltetralone lignans proposed by Müller and Vajda (1952).

\section{Results and discussion}

The acetone extract of $H$. reniformis roots was subjected to column chromatography followed by HPLC to yield two minor new lignans (1 and 2, Fig. 1) together with the known compounds (-)-futokadsurin C (3) and (-)-8'-epi-aristoligone (4a). They were analyzed by spectroscopic methods, and the known compounds 


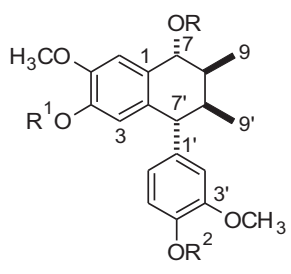

$$
\begin{array}{llll} 
& \mathbf{R} & \mathbf{R}^{1} & \mathbf{R}^{\mathbf{2}} \\
\mathbf{1} & \mathrm{H} & \mathrm{H} & \mathrm{CH}_{3} \\
\mathbf{2} & \mathrm{CH}_{3} & \mathrm{H} & \mathrm{CH}_{3} \\
\mathbf{5} & \mathrm{H} & \mathrm{CH}_{3} & \mathrm{CH}_{3} \\
\mathbf{6} & \mathrm{CH}_{3} & \mathrm{CH}_{3} & \mathrm{CH}_{3} \\
\mathbf{7} & \mathrm{CH}_{3} & \mathrm{CH}_{3} & \mathrm{H}
\end{array}
$$<smiles>COc1ccc([C@@H]2O[C@@H](c3ccc4c(c3)OCO4)[C@H](C)[C@H]2C)cc1OC</smiles>

3<smiles>COc1ccc([C@H]2c3cc(OC)c(OC)cc3C(=O)C(C)[C@@H]2C)cc1OC</smiles>

$4 a$

Fig. 1. Chemical structures of compounds 1-3, 4a, and 5-7.

were identified by comparison with data reported in the literature (da Silva and Lopes, 2004, 2006; Konishi et al., 2005; Kuo et al., 1989) (Fig. 1).

The ${ }^{1} \mathrm{H}$ and ${ }^{13} \mathrm{C}$ NMR, IR, and UV spectroscopic data for compounds $\mathbf{1}$ and $\mathbf{2}$ were very similar to those reported for the aryltetralol derivatives, previously isolated from $H$. reniformis and obtained by microbial transformation of the aryltetralin lignans 5-7 (da Silva and Lopes, 2006; Messiano et al., 2008, 2010). Compounds $\mathbf{1}$ and $\mathbf{2}$ were also suggested to be aryltetralol lignans based on the HRMS spectra. They displayed quasi-molecular ions at $m / z 381.1663[\mathrm{M}+\mathrm{Na}]^{+}$and at $m / z 371.1445[\mathrm{M}-\mathrm{H}]^{-}$, respectively, which were consistent with the molecular formula $\mathrm{C}_{21} \mathrm{H}_{26} \mathrm{O}_{5}$ for 1 and $\mathrm{C}_{22} \mathrm{H}_{28} \mathrm{O}_{5}$ for $2(14 \mu$ higher than 1). The IR spectra of both compounds showed characteristic absorptions of hydroxyl groups at $\sim 3440 \mathrm{~cm}^{-1}$. The ${ }^{1} \mathrm{H}$ and ${ }^{13} \mathrm{C}$ NMR spectra of both compounds (Tables 1 and 2, Figs. S1-S4) suggested the presence of a veratryl group ( $C$ ring), and one methoxyl group linked to 1,2,4,5tetrasubstituted aromatic ring (A). These spectra also showed signals for two methyl groups and four methine carbons, of which one is oxygenated. In addition, signals for hydroxyl groups at $\delta_{\mathrm{H}} \sim 5.4$ were observed. Compound 2 showed additional resonances reminiscent of an aliphatic methoxyl group $\left(\delta_{\mathrm{C}} 56.0\right.$; $\delta_{\mathrm{H}}$ 3.40). The differences between the chemical shifts of carbons and hydrogens on the $\mathrm{B}$ ring of $\mathbf{1}$ and $\mathbf{2}$, particularly of $\mathrm{CH}-7$
(1: $\left.\delta_{\mathrm{C}} 74.1 ; \delta_{\mathrm{H}} 4.38 ; 2: \delta_{\mathrm{C}} 83.4 ; \delta_{\mathrm{H}} 3.92\right)$ and $\mathrm{CH}-8\left(\mathbf{1}: \delta_{\mathrm{C}} 39.4\right.$; $\left.\delta_{\mathrm{H}} 1.99 ; 2: \delta_{\mathrm{C}} 34.6 ; \delta_{\mathrm{H}} 2.19\right)$, suggested that these compounds differ by the presence of a methoxyl group at C-7 in $\mathbf{2}$ instead of a hydroxyl group in 1. Furthermore, gHMBC experiments supported correlations between $\mathrm{C}-7$ and $\mathrm{OCH}_{3}-7, \mathrm{H}-6$, and $3 \mathrm{H}-9$. The magnitude of the coupling constants of the methine hydrogens in the $\mathrm{B}$ ring evidenced trans diaxial, cis axial-equatorial, and trans diequatorial positions for $\mathrm{H}-7^{\prime}, 8^{\prime} \quad(J=10.0 \pm 0.5 \mathrm{~Hz}), \quad \mathrm{H}-8^{\prime}, 8$ $(J=3.5 \mathrm{~Hz})$, and $\mathrm{H}-7,8(J=3.7 \pm 0.3 \mathrm{~Hz})$, respectively. Moreover, gNOESY experiments showed spatial interactions between $\mathrm{H}-7$ and $3 \mathrm{H}-9$, as well as between $\mathrm{H}-7^{\prime}$ and $\mathrm{H}-3, \mathrm{H}-2^{\prime}, \mathrm{H}-6^{\prime}, 3 \mathrm{H}-9$, and $3 \mathrm{H}-9^{\prime}$ for both compounds. Furthermore, spatial interactions of $\mathrm{H}-6(\delta 6.68)$ with $\mathrm{H}-7, \mathrm{OCH}_{3}-7(\delta 3.40)$, and $\mathrm{OCH}_{3}-5(\delta 3.84)$ were observed in gNOESY experiments of $\mathbf{2}$. Both compounds showed very similar CD curves with a positive Cotton effect at $\lambda \sim 290 \mathrm{~nm}$, consistent with a $7^{\prime} R$ configuration (da Silva et al., 2004). Thus, the absolute configuration of $\mathbf{1}$ and $\mathbf{2}$ was determined as $\left(7 R, 7^{\prime} R, 8 S, 8^{\prime} S\right)$, which was identical to that previously determined for 5-7 (da Silva and Lopes, 2006; Messiano et al., 2010).

The synthesis of aryltetralone (4 and 11), aryltetralol (12), and aryltetralene (13) lignans was achieved from four to six steps,

\begin{tabular}{|c|c|c|c|c|}
\hline \multirow{2}{*}{$\begin{array}{l}\text { Position } \\
\mathrm{H}\end{array}$} & \multicolumn{2}{|l|}{1} & \multicolumn{2}{|l|}{2} \\
\hline & $\delta_{\mathrm{H}}(\mathrm{J} \text { in } \mathrm{Hz})^{\mathrm{a}}$ & gNOESY & $\delta_{\mathrm{H}}(\mathrm{J} \text { in } \mathrm{Hz})^{\mathrm{a}}$ & gNOESY \\
\hline 3 & $6.28, \mathrm{~d}(1.0)$ & $7^{\prime}$ & $6.29, \mathrm{~d}(0.5)$ & $7^{\prime}$ \\
\hline 6 & $6.78, \mathrm{~s}$ & $7, \mathrm{OCH}_{3}-5$ & $6.68, \mathrm{~s}$ & $7, \mathrm{OCH}_{3}-5, \mathrm{OCH}_{3}-7$ \\
\hline 7 & $4.38, \mathrm{~d}(4.0)$ & $6,8,9$ & $3.92, \mathrm{~d}(3.5)$ & $6,8,9, \mathrm{OCH}_{3}-7$ \\
\hline 8 & 1.99 , ddq $(4.0,3.5,7.5)$ & $7,9,8^{\prime}, 9^{\prime}$ & 2.19, ddq $(3.5,3.5,7.0)$ & $7,9,8^{\prime}, \mathrm{OCH}_{3}-7$ \\
\hline 9 & $0.83, \mathrm{~d}(7.5)$ & $7,8,7^{\prime}, 9^{\prime}$ & $0.79, \mathrm{~d}(7.0)$ & $7,8,7^{\prime}, 9^{\prime}$ \\
\hline $2^{\prime}$ & $6.55, \mathrm{~d}(2.0)$ & $7^{\prime}, 8^{\prime}, \mathrm{OCH}_{3}-3^{\prime}$ & $6.55, \mathrm{~d}(2.0)$ & $7^{\prime}, 8^{\prime}, \mathrm{OCH}_{3}-3^{\prime}$ \\
\hline $5^{\prime}$ & $6.70, \mathrm{~d}(8.0)$ & $\mathrm{OCH}_{3}-4^{\prime}$ & $6.71, \mathrm{~d}(8.0)$ & $\mathrm{OCH}_{3}-4^{\prime}$ \\
\hline $6^{\prime}$ & 6.56 , dd $(8.0,2.0)$ & $5^{\prime}, 7^{\prime}, 8^{\prime}$ & 6.63, dd $(8.0,2.0)$ & $7^{\prime}$ \\
\hline $7^{\prime}$ & 3.36, br d (9.5) & $3,9,2^{\prime}, 6^{\prime}, 9^{\prime}$ & 3.30, br d (10.5) & $3,9,2^{\prime}, 6^{\prime}, 9^{\prime}$ \\
\hline $8^{\prime}$ & 2.32 , ddq $(9.5,3.5,7.0)$ & $8,2^{\prime}, 6^{\prime}, 9^{\prime}$ & $2.35, \mathrm{ddq},(10.5,3.5,7.0)$ & $8,2^{\prime}, 9^{\prime}$ \\
\hline $9^{\prime}$ & $0.81, \mathrm{~d}(7.0)$ & $8,9,8^{\prime}$ & $0.81, \mathrm{~d}(7.0)$ & $8^{\prime}$ \\
\hline $\mathrm{OCH}_{3}-3^{\prime}$ & $3.73, \mathrm{~s}$ & $2^{\prime}$ & $3.73, \mathrm{~s}$ & \\
\hline $\mathrm{OCH}_{3}-4^{\prime}$ & $3.78, \mathrm{~s}$ & $5^{\prime}$ & $3.80, \mathrm{~s}$ & \\
\hline $\mathrm{OCH}_{3}-5$ & $3.81, \mathrm{~s}$ & 6 & $3.84, \mathrm{~s}$ & \\
\hline $\mathrm{OCH}_{3}-7$ & & & $3.40, \mathrm{~s}$ & 7,8 \\
\hline $\mathrm{OH}-4$ & 5.49 , br s & & 5.37 , br s & \\
\hline
\end{tabular}
successively, starting from stable and easily available materials. Improving the scheme proposed by Müller and Vajda (1952), via Reformatsky, by using veratrylacetone and $\alpha$-bromopropianate,

Table 1

${ }^{1} \mathrm{H}$ NMR spectroscopic data for compounds 1 and $\mathbf{2}\left(\mathrm{CDCl}_{3}, 11.7 \mathrm{~T}\right)$.

\footnotetext{
${ }^{\text {a }}$ Multiplicities were determined with the assistance of ${ }^{1} \mathrm{H}^{-1} \mathrm{H}$ COSY spectra and simulation using ACD/C $+{ }^{1} \mathrm{H}$ NMR predictors $(\mathrm{ACD}, 2010)$.
} 
Table 2

${ }^{13} \mathrm{C}$ NMR spectroscopic data for compounds $\mathbf{1}$ and $\mathbf{2}\left(\mathrm{CDCl}_{3}, 11.7 \mathrm{~T}\right)$.

\begin{tabular}{|c|c|c|c|c|}
\hline \multirow{2}{*}{$\begin{array}{l}\text { Position } \\
\mathrm{H} \\
\end{array}$} & \multicolumn{2}{|l|}{1} & \multicolumn{2}{|l|}{2} \\
\hline & $\delta_{\mathrm{C}}$, type $^{\mathrm{a}}$ & gHMBC $^{b}$ & $\delta_{\mathrm{C}}$, type $^{\mathrm{a}}$ & gHMBC $^{\text {b }}$ \\
\hline 1 & 128.6, C & $\mathrm{H}-3,7,7^{\prime}$ & $126.0, \mathrm{C}$ & $\mathrm{H}-3$ \\
\hline 2 & 133.0, C & $\mathrm{H}-6,7,7^{\prime}$ & 134.0, C & H-6 \\
\hline 3 & 115.6, CH & $\mathrm{H}-7^{\prime}$ & $115.5, \mathrm{CH}$ & \\
\hline 4 & $145.3, \mathrm{C}$ & $\mathrm{H}-3$ and/or H-6 & $145.3, \mathrm{C}$ & H-6 \\
\hline 5 & $145.4, \mathrm{C}$ & $\mathrm{H}-3$ and/or $\mathrm{H}-6, \mathrm{OCH}_{3}-5$ & 144.9, C & $\mathrm{H}-3, \mathrm{OCH}_{3}-5$ \\
\hline 6 & $111.4, \mathrm{CH}$ & $\mathrm{H}-7$ & $112.2, \mathrm{CH}$ & $\mathrm{H}-7$ \\
\hline 7 & 74.1, CH & $\mathrm{H}-9$ & $83.4, \mathrm{CH}$ & $\mathrm{H}-6,9, \mathrm{OCH}_{3}-7$ \\
\hline 8 & $39.4, \mathrm{CH}$ & $\mathrm{H}-9$ & $34.6, \mathrm{CH}$ & $\mathrm{H}-7^{\prime}, 9^{\prime}$ \\
\hline 9 & 11.7, $\mathrm{CH}_{3}$ & $\mathrm{H}-7$ & $11.1, \mathrm{CH}_{3}$ & \\
\hline $1^{\prime}$ & 138.1, C & $\mathrm{H}-5^{\prime}, 7^{\prime}$ & $138.4, \mathrm{C}$ & $\mathrm{H}-5^{\prime}$ \\
\hline $2^{\prime}$ & $112.4, \mathrm{CH}$ & $\mathrm{H}-6^{\prime}, 7^{\prime}$ & $112.2, \mathrm{CH}$ & $\mathrm{H}-6^{\prime}, \mathrm{H}-\mathrm{7}^{\prime \prime}$ \\
\hline $3^{\prime}$ & 148.9, C & $\mathrm{H}-5^{\prime}, \mathrm{OCH}_{3}-3^{\prime}$ & $148.9, \mathrm{C}$ & $\mathrm{H}-5^{\prime}, \mathrm{OCH}_{3}-3^{\prime}$ \\
\hline $4^{\prime}$ & 147.5, C & $\mathrm{H}-2^{\prime}, 6^{\prime}, \mathrm{OCH}_{3}-4^{\prime}$ & $147.4, \mathrm{C}$ & $\mathrm{H}-2^{\prime}, 6^{\prime}, \mathrm{OCH}_{3}-4^{\prime}$ \\
\hline $5^{\prime}$ & $111.0, \mathrm{CH}$ & & $110.8, \mathrm{CH}$ & \\
\hline $6^{\prime}$ & 121.7, CH & $\mathrm{H}-2^{\prime}, 5^{\prime}, 7^{\prime}$ & $121.8, \mathrm{CH}$ & $\mathrm{H}-2^{\prime}$ \\
\hline $7^{\prime}$ & 48.7, $\mathrm{CH}$ & $\mathrm{H}-3,2^{\prime}, 6^{\prime}, 9^{\prime}$ & $48.8, \mathrm{CH}$ & $\mathrm{H}-3,2^{\prime}, 9^{\prime}$ \\
\hline $8^{\prime}$ & $35.1, \mathrm{CH}$ & $\mathrm{H}-7,9,7^{\prime}$ & $35.3, \mathrm{CH}$ & \\
\hline $9^{\prime}$ & $16.8, \mathrm{CH}_{3}$ & $\mathrm{H}-7^{\prime}$ & $17.2, \mathrm{CH}_{3}$ & $\mathrm{H}-7^{\prime}$ \\
\hline $\mathrm{OCH}_{3}-3^{\prime}$ & $55.9, \mathrm{CH}_{3}$ & & $56.0, \mathrm{CH}_{3}$ & \\
\hline $\mathrm{OCH}_{3}-4^{\prime}$ & $55.9, \mathrm{CH}_{3}$ & & $55.9, \mathrm{CH}_{3}$ & \\
\hline $\mathrm{OCH}_{3}-5$ & $55.9, \mathrm{CH}_{3}$ & & $55.9, \mathrm{CH}_{3}$ & \\
\hline $\mathrm{OCH}_{3}-7$ & & & $56.0, \mathrm{CH}_{3}$ & \\
\hline
\end{tabular}

a Chemical shifts and multiplicities were determined with the assistance of DEPT and gHMQC experiments.

${ }^{\mathrm{b}}$ gHMBC correlations, optimized for $8 \mathrm{~Hz}$, are from carbon stated to the indicated hydrogen(s).

two pairs of aryltetralone enantiomers $(\mathbf{4}+\mathbf{1 1})$ were obtained, after HPLC separation, in four steps with good yield (87.5\%). The major bottleneck in $\mathbf{4 + 1 1}$ synthesis was the lactonization after Reformatsky reaction. By extracting the intermediate lactone (9) with $\mathrm{CHCl}_{3}$ instead of distilling it (Müller and Vajda, 1952), the yield of this step increased from $28 \%$ to $99.8 \%$.

After separation by HPLC, each pair of enantiomers (4 and 11) was evaluated in vitro for their antiplasmodial activity. The immunoenzymatic test with monoclonal antibodies to the parasite protein histidine- and alanine-rich (HRPII), as shown in SI, was used to assess the inhibition of growth of $P$. falciparum in the presence of these compounds (Krettli et al., 2009; Noedl et al., 2002). Enantiomers $\mathbf{1 1}$ and enantiomers $\mathbf{4}$ showed to be inactive $\left(\mathrm{IC}_{50} \geq 82 \mu \mathrm{M}\right.$, Table S1), suggesting that the stereochemistry is very important for the antiplasmodial activity of this type of lignans. Aiming to obtain pure enantiomers to advance in the biological tests and chemical transformations, to evaluate the importance of C-7 carbonyl group for the activity, samples of $\mathbf{4}$ were analyzed by chiral-HPLC by using several conditions in analytical scales (columns, solvents and flows). Unfortunately, no suitable condition to separate the enantiomers 4 in a semipreparative scale was achieved. Thus, samples of $\mathbf{4 + 1 1}$ and the natural aryltetralone $\mathbf{4 a}\left[(-)-8^{\prime}\right.$-epi-aristoligone $]$ were individually subjected to reduction with $\mathrm{NaBH}_{4}$ in methanol to give 12 and 12a, respectively. The structure of $\mathbf{1 2 a}$ was confirmed by comparison of its 1D and 2D NMR spectra and optical activity $\left([\alpha]_{\mathrm{D}}=-27\left(\mathrm{CHCl}_{3}\right.\right.$, $c$ 0.10)) with those of an authentic sample of (-)-aristoligol (12a) $[\alpha]_{\mathrm{D}}=-20\left(\mathrm{CHCl}_{3}, c 0.10\right)$ (da Silva and Lopes, 2006). Interestingly, the stereochemistry of $\mathrm{C}-\mathbf{7}$ of $\mathbf{1 2 a}$ is different from that of the natural aryltetralol (1). Finally, subjecting 12 and 12a to dehydration with $p$-toluenesulfonic acid, the products 13 and 13a were obtained in $83.3 \%$ and $85 \%$ yield, respectively (Fig. 2).

A better chromatographic condition, with lower $t_{R}$, was achieved for the separation of the enantiomers $\mathbf{1 3}$ than for the aryltetralones 4 using chiral columns (Fig. S5). Thus, 13 was subjected to semi-preparative chiral-HPLC to give (-)-cyclogalgravin 13a $\left(t_{R}=14.3 \mathrm{~min}, 50.9 \%\right)$ and (+)-cyclogalgravin 13b $\left(t_{\mathrm{R}}=13.4 \mathrm{~min}, 49.1 \%\right)$.
The similarity of the ${ }^{1} \mathrm{H}$ and ${ }^{13} \mathrm{C}$ NMR data and optical activities of 13a $\left([\alpha]_{D}=-96\left(\mathrm{CHCl}_{3} ; c 0.21\right),[\theta]_{290}-10330\right)$ with those from the natural product $\left([\alpha]_{\mathrm{D}}=-106\left(\mathrm{CHCl}_{3} ; c 1.07\right),[\theta]_{290}-1254\right)$ (da Silva and Lopes, 2006) confirmed the assigned relative and absolute configurations for 13a.

The compound 12a and 13a exhibited the highest activity ( IC $_{50} 8.4$ and $10.8 \mu \mathrm{M}$, respectively), whereas $\mathbf{1 3 b}$ showed moderate activity ( IC $_{50} 30 \mu \mathrm{M}$ ) (Table S1). The natural aryltetralols $\mathbf{1}$ and $\mathbf{5}$ also showed moderate activity $\left(\mathrm{IC}_{50} 33.6\right.$ and $33.1 \mu \mathrm{M}$, respectively), while the $\mathrm{C}-7 \mathrm{OCH}_{3}$ analogous $\mathbf{2}$ and $\mathbf{6}$ were inactive (Table S1). These results suggest that steric effects also have influence on the activity. Among the tested lignans, 12a and 13a exhibited good selectivity index $(\mathrm{SI}>28)$.

\section{Experimental}

\subsection{General experimental procedures}

One-dimensional $\left({ }^{1} \mathrm{H},{ }^{13} \mathrm{C}\right.$, DEPT, HOMODEC, TOCSY, and gNOESY) and two-dimensional $\left({ }^{1} \mathrm{H}-{ }^{1} \mathrm{H}\right.$ gCOSY, gNOESY, gHMQC, gHSQC, and gHMBC) NMR experiments were performed on a Varian INOVA 500 spectrometer $(11.7 \mathrm{~T})$ at $500 \mathrm{MHz}\left({ }^{1} \mathrm{H}\right)$, and $126 \mathrm{MHz}\left({ }^{13} \mathrm{C}\right)$, using deuterated solvents $\left(\mathrm{CDCl}_{3}\right.$ and DMSO- $\left.\mathrm{d}_{6}\right)(\mathrm{P}$ 99.9\% D) as an internal standards for ${ }^{13} \mathrm{C}$ NMR chemical shifts and residual solvent as an internal standard for ${ }^{1} \mathrm{H}$ NMR. $\delta$ values are reported relative to TMS. Mass spectra (ESI-MS) were obtained on a LCQ Fleet-Thermo Scientific, and flow injection into the electrospray source was used for LC-ESI-MS. High-resolution mass spectra (HRMS) were obtained on a Bruker Daltonics ultrOTOFq (ESITOFMS). IR spectra were obtained on a PerkinElmer 1600 FT-IR spectrometer using $\mathrm{KBr}$ discs. Optical rotations were measured on a PerkinElmer 341-LC polarimeter. Ultraviolet (UV) absorptions were measured on a PerkinElmer UV-vis Lambda 14P diode array spectrophotometer. Circular dichroism (CD) spectra were recorded on a JASCO J-815 spectrometer, using $0.2 \mathrm{~mm}$ cell. HPLC analyses were performed using a Shimadzu liquid chromatograph (SPD-10 Avp), equipped with UV-vis and 341-LC polarimeter detectors, and using a Jasco LC-NetII/ADC, equipped with photodiode array (MD2018 Plus) and CD (2095 Plus) detectors, and chromatograms were acquired at $270 \mathrm{~nm}$ and $254 \mathrm{~nm}$. The columns RP-18 (C18, Varian), Chiralpak ${ }^{\circledR}$ IC (DAICEL), Lux $5 \mu$ Cellulose-1 (Phenomenex ${ }^{\circledR}$ ), and $\beta$-CD BR Chiralpak ${ }^{\circledR}$ (YMC) having a particle size of $5 \mu \mathrm{m}$, were used for analytical analysis $(250 \times 4.6 \mathrm{~mm})$ and for semipreparative analysis $(250 \times 10 \mathrm{~mm})$. All reactions were monitored by TLC using $0.25 \mathrm{~mm}$ E. Merk silica gel plates $\left(60 \mathrm{PF}_{254}\right)$ with $\mathrm{UV}, \mathrm{I}_{2}$ vapor, or $10 \% \mathrm{H}_{2} \mathrm{SO}_{4}$-heat as developing agent. All reactions were carried out under $\mathrm{N}_{2}$ atmosphere with freshly distilled solvents under anhydrous conditions unless otherwise noted. Reagents were purchased from Sigma-Aldrich Co., and used without purification, except where noted. Solvents employed were HPLC grade from Mallinckrodt. All yields refer to chromatographically and spectroscopically $\left({ }^{1} \mathrm{H}\right.$ and ${ }^{13} \mathrm{C} N \mathrm{NM}, \alpha_{\mathrm{D}}$ ) homogenous material unless otherwise stated. Ultrapure water was obtained from Milli-Q Gradient A10 from Millipore.

\subsection{Plant materials}

The plant materials were collected in Ituiutaba, MG, Brazil, in February 2010, and identified as Holostylis reniformis Duch. by Dr. Vinícius C. Souza and Dr. Lindolpho Cappellari Jr. A voucher specimen (ESA 110,744/2010) was deposited at the herbarium of the Escola Superior de Agricultura, Luiz de Queiroz (ESALQ), Piracicaba, SP, Brazil. Authorization CGEN/MMA number 10,586/ 2012-1. The material was separated according to the plant parts and dried $\left(\sim 45^{\circ} \mathrm{C}\right)$. 
併<smiles>CCOC(=O)C(C)C(C)(O)Cc1ccc(OC)c(OC)c1</smiles><smiles>COc1ccc(C(c2ccc(OC)c(OC)c2)C(C)C(C)C(=O)O)cc1OC</smiles>

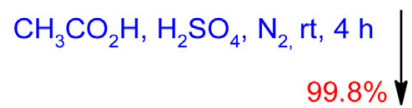

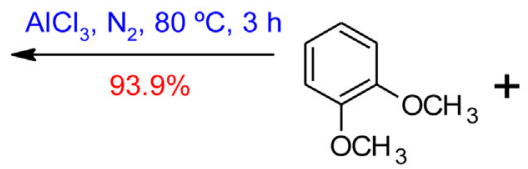
$\mathrm{PCl}_{5}, \mathrm{SnCl}_{4}, \mathrm{C}_{6} \mathrm{H}_{6}, \mathrm{~N}_{2}, 0^{\circ} \mathrm{C}, 0.5 \mathrm{~h}$ $95.1 \%$<smiles>COc1ccc([C@H]2c3cc(OC)c(OC)cc3C(=O)[C@H](C)[C@@H]2C)cc1OC</smiles>
$4[(-)-4 a+(+)-4 b]$

<smiles>COc1ccc([C@H]2c3cc(OC)c(OC)cc3C(=O)[C@@H](C)[C@@H]2C)cc1OC</smiles>
$11[(-)-11 a+(+)-11 b]$

$12\left[(-)-12-\mathbf{a}: \beta O H, \beta \mathrm{CH}_{3}\right]$<smiles>COc1cc2c(cc1OC)[C@H](O)[C@H](C)[C@@H](C)[C@@H]2C</smiles><smiles>COc1ccccc1OC</smiles>

\begin{tabular}{|l|l|}
\hline & $\begin{array}{l}\text { i) } \mathrm{TsOH}, \mathrm{C}_{6} \mathrm{H}_{6}, \Delta \\
\text { ii) Chiral-HPLC }\end{array}$ \\
\hline $83.3 \%$
\end{tabular}<smiles>COc1ccc([C@H]2c3cc(OC)c(OC)cc3C=C(C)[C@@H]2C)cc1OC</smiles><smiles>COc1ccc([C@H]2c3cc(OC)c(OC)cc3C=C(C)[C@@H]2C)cc1OC</smiles>

$(-)-13 a$

$(+)-13 b$

Fig. 2. Total syntheses of aryltetralenes 13a and $\mathbf{1 3 b}$. 


\subsection{Extraction and isolation of the chemical constituents}

The roots $(3.7 \mathrm{~kg}$ ) were ground and exhaustively extracted successively at room temperature with hexanes, acetone, and ethanol [ $4 \times(\sim 200 \mathrm{~mL}, 2$ days and shaken manually every $12 \mathrm{~h}$ for $2 \mathrm{~min}$ ) each solvent] (da Silva and Lopes, 2006; Messiano et al., 2009). The residues were extracted with ethanol in a Soxhlet apparatus and the extracts were individually concentrated.

The crude acetone extract $(6.6 \mathrm{~g})$ was subjected to CC $(40.0 \times 5.0 \mathrm{~cm}$, silica gel $60 \mathrm{H}, 203.5 \mathrm{~g}, n$-hexanes/EtOAc gradient, $19: 1$ to $100 \%$ EtOAc) to give 31 fractions (ca. $120 \mathrm{~mL}$ each), as previously described (da Silva et al., 2004). After semi-preparative $\mathrm{HPLC}\left(\mathrm{C} 18, \mathrm{MeOH} / \mathrm{H}_{2} \mathrm{O}, 7: 3\right.$, flow rate: $\left.8 \mathrm{~mL} / \mathrm{min}\right)$ fractions 16 and 19 gave $3(8.2 \mathrm{mg})$ and $\mathbf{4 a}(1140.0 \mathrm{mg})$, respectively. Fraction 23 $(280.0 \mathrm{mg})$ gave $\mathbf{1}(43.5 \mathrm{mg})$ and $\mathbf{2}(8.9 \mathrm{mg})$ after semi-preparative HPLC (C18, $\mathrm{MeOH} / \mathrm{H}_{2} \mathrm{O}, 3: 2$, flow rate: $8 \mathrm{~mL} / \mathrm{min}$ ).

3.3.1. (-)-(7R, $\left.7^{\prime} \mathrm{R}, 8 \mathrm{~S}, 8^{\prime} \mathrm{S}\right)-4,7-D i h y d r o x y-3^{\prime}, 4^{\prime}, 5$-trimethoxy-2,7'cyclolignan [(-)-4-0-demethyl-7-hydroxyisogalbulin, 1]

Yellow oil $\left(\mathrm{CHCl}_{3}\right) ;[\alpha]_{\mathrm{D}}^{25}-38\left(c \mathrm{0.7}, \mathrm{CHCl}_{3}\right)$; UV $\lambda{ }_{\max }^{\mathrm{MeOH}}$ see Fig. S6; IR (KBr) $v_{\max } 3434,1590,1513,1463 \mathrm{~cm}^{-1} ;{ }^{1} \mathrm{H}$ and ${ }^{13} \mathrm{C} \mathrm{NMR}\left(\mathrm{CDCl}_{3}\right)$ see Tables 1 and 2; ESIMS $20 \mathrm{eV}$, positive mode, $m / z$ (rel. int.): 359 $[\mathrm{M}+\mathrm{H}]^{+}$(100); HRESIMS (probe) $4.5 \mathrm{eV}$, positive mode, $\mathrm{m} / \mathrm{z}$ (rel. int.): $381.1663[\mathrm{M}+\mathrm{Na}]^{+}$(100) (calcd for $\mathrm{C}_{21} \mathrm{H}_{26} \mathrm{O}_{5} \mathrm{Na}$, 381.1672); $\mathrm{CD}\left(\mathrm{CHCl}_{3}\right.$, c 0.50$):[\theta]_{307}-5626,[\theta]_{296}+22895$, $[\theta]_{279}-44311,[\theta]_{260}-14739,[\theta]_{241}-141569$.

\subsection{2. (7R, $\left.7^{\prime} \mathrm{R}, 8 \mathrm{~S}, 8^{\prime} \mathrm{S}\right)$-4-Hydroxy-3',4',5,7-tetramethoxy-2,7' -} cyclolignan [(-)-4-0-demethyl-7-methoxyisogalbulin, 2]

Yellow oil $\left(\mathrm{CHCl}_{3}\right) ;[\alpha]_{\mathrm{D}}^{25}-26\left(c \mathrm{0.6}, \mathrm{CHCl}_{3}\right)$; UV $\lambda_{\max }^{\mathrm{MeOH}}$ see Fig. S6; $\operatorname{IR}(\mathrm{KBr}) v_{\max } 3446,1590,1520,1430 \mathrm{~cm}^{-1} ;{ }^{1} \mathrm{H}$ and ${ }^{13} \mathrm{C} \mathrm{NMR}\left(\mathrm{CDCl}_{3}\right)$ see Tables 1 and 2; HRESIMS (probe) $4.5 \mathrm{eV}$, negative mode, $\mathrm{m} / \mathrm{z}$ (rel. int.): $371.1445[\mathrm{M}-\mathrm{H}]^{-}$(100) (calcd for $\mathrm{C}_{22} \mathrm{H}_{27} \mathrm{O}_{5}, 371.1853$ ); $\mathrm{CD}\left(\mathrm{CHCl}_{3}, c\right.$ 0.57): $[\theta]_{297}+5494,[\theta]_{278}-18353,[\theta]_{245}-113950$.

\subsection{3. (7R, $\left.7^{\prime} \mathrm{R}, 8 \mathrm{~S}, 8^{\prime} \mathrm{S}\right)$-7-Hydroxy-3',4',4,5-tetramethoxy-2,7'-} cyclolignan [(-)-holostylol, 12a]

Yellow oil $\left(\mathrm{CHCl}_{3}\right) ;[\alpha]_{\mathrm{D}}^{25}-28\left(c 0.10, \mathrm{CHCl}_{3}\right)$ lit. (da Silva et al., 2006) $[\alpha]_{\mathrm{D}}^{25}-20$ (c 1.6, $\left.\mathrm{CHCl}_{3}\right) .{ }^{1} \mathrm{H}$ NMR $\left(\mathrm{CDCl}_{3}, 500 \mathrm{MHz}\right): \delta 6.16$ $(1 \mathrm{H}, \mathrm{s}, \mathrm{H}-3), 7.08(1 \mathrm{H}, \mathrm{s}, \mathrm{H}-6), 4.93(1 \mathrm{H}, \mathrm{d}, J=4.5 \mathrm{~Hz}, \mathrm{H}-7), 2.16(1 \mathrm{H}$, ddq, $J=3.0,4.5,7.0 \mathrm{~Hz}, \mathrm{H}-8), 0.86(3 \mathrm{H}, \mathrm{d}, J=7.0 \mathrm{~Hz}, \mathrm{H}-9), 6.48(1 \mathrm{H}, \mathrm{d}$, $\left.J=2.0 \mathrm{~Hz}, \mathrm{H}-2^{\prime}\right), 6.72\left(1 \mathrm{H}, \mathrm{d}, J=8.0 \mathrm{~Hz}, \mathrm{H}-5^{\prime}\right), 6.58(1 \mathrm{H}, \mathrm{dd}, J=8.0$, $\left.2.0 \mathrm{~Hz}, \mathrm{H}-6^{\prime}\right), 3.48\left(1 \mathrm{H}, \mathrm{d}, J=10.0 \mathrm{~Hz}, \mathrm{H}-7^{\prime}\right), 2.01(1 \mathrm{H}, \mathrm{ddq}, J=3.0$, $\left.10.0,7.0 \mathrm{~Hz}, \mathrm{H}-8^{\prime}\right), 0.84\left(3 \mathrm{H}, \mathrm{d}, J=7.0 \mathrm{~Hz}, \mathrm{H}-9^{\prime}\right), 3.54\left(\mathrm{~s}, \mathrm{OCH}_{3}-4\right), 3.84$ (s, $\left.\mathrm{OCH}_{3}-5\right), 3.74\left(\mathrm{~s}, \mathrm{OCH}_{3}-3^{\prime}\right), 3.81\left(\mathrm{~s}, \mathrm{OCH}_{3}-4^{\prime}\right)$.

\subsection{4. ( $\left.7^{\prime} \mathrm{R}, 8^{\prime} \mathrm{S}\right)-3^{\prime}, 4^{\prime}, 4,5$-Tetramethoxy-2,7'-cyclolignan-7-eno}

[(-)-cyclogalgravin, 13a]

Yellow oil $\left(\mathrm{CHCl}_{3}\right) ;[\alpha]_{\mathrm{D}}^{25}-103\left(c \mathrm{0.10}, \mathrm{CHCl}_{3}\right)$, lit. (da Silva et al., 2006) $[\alpha]_{\mathrm{D}}^{25}-106$ (c 1.07, $\left.\mathrm{CHCl}_{3}\right) .{ }^{1} \mathrm{H} \mathrm{NMR}\left(\mathrm{CDCl}_{3}, 300 \mathrm{MHz}\right): \delta 6.49$ $(1 \mathrm{H}, \mathrm{s}, \mathrm{H}-3), 6.56(1 \mathrm{H}, \mathrm{s}, \mathrm{H}-6), 6.08(1 \mathrm{H}, \mathrm{br} \mathrm{s}, \mathrm{H}-7), 1.73(3 \mathrm{H}, \mathrm{d}$, $J=1,2 \mathrm{~Hz}, \mathrm{H}-9), 6.60\left(1 \mathrm{H}, \mathrm{d}, J=2.1 \mathrm{~Hz}, \mathrm{H}-2^{\prime}\right), 6.65(1 \mathrm{H}, \mathrm{d}, J=8.4 \mathrm{~Hz}, \mathrm{H}-$ 5'), $6.49\left(1 \mathrm{H}, \mathrm{dd}, J=8.4,2.1 \mathrm{~Hz}, \mathrm{H}-6^{\prime}\right), 3.62\left(1 \mathrm{H}, \mathrm{d}, J=3.3 \mathrm{~Hz}, \mathrm{H}-7^{\prime}\right)$, $2.33\left(1 \mathrm{H}, \mathrm{dq}, J=7.2,3.3 \mathrm{~Hz}, \mathrm{H}-8^{\prime}\right), 1.02\left(3 \mathrm{H}, \mathrm{d}, J=7.2 \mathrm{~Hz}, \mathrm{H}-9^{\prime}\right), 3.76(\mathrm{~s}$, $\left.\mathrm{OCH}_{3}-4\right)$, $3.82\left(\mathrm{~s}, \mathrm{OCH}_{3}-5\right)$; $3.72\left(\mathrm{~s}, \mathrm{OCH}_{3}-3^{\prime}\right)$, $3.72\left(\mathrm{~s}, \mathrm{OCH}_{3}-4^{\prime}\right)$.

\subsection{Syntheses and chemical transformations}

\subsubsection{Benzenebutanoic acid, $\beta$-hydroxy-3,4-dimethoxy-}

$\alpha, \beta$-dimethyl-, ethyl ester (8):

$\alpha$-Bromopropionate $(2.89 \mathrm{~g}, 17.4 \mathrm{mmol}$ ) was dropwise added to a stirred solution of veratrylacetone $(3.08 \mathrm{~g}, 17.2 \mathrm{mmol})$ in $9 \mathrm{~mL}$ of dry benzene (under $\mathrm{N}_{2}$ ) and zinc (previously washed with acetone and activated at $100^{\circ} \mathrm{C}$ for $8 \mathrm{~h}$ (Chavan, 2004)). The stirring solution was heated until the reflux began, this being maintained for $4 \mathrm{~h}$. Then, the mixture was cooled and the ester $(4.24 \mathrm{~g}$, $16.89 \mathrm{mmol}, 98.2 \%)$ extracted with $\mathrm{CHCl}_{3}(3 \times 50 \mathrm{~mL})$.

\subsection{2. $\alpha, \beta$-Dimethyl- $\gamma$-(3,4-dimethoxyphenyl) butyrolactone (9)}

The ester ( $3.92 \mathrm{~g}, 15.6 \mathrm{mmol})$ was dissolved in $\mathrm{CH}_{3} \mathrm{CO}_{2} \mathrm{H}(13 \mathrm{~mL})$ and then ice-cooling. To this cooling and stirring solution sulfuric acid $(3 \mathrm{~mL})$ was added by drops over $1 \mathrm{~h}$. After $4 \mathrm{~h}$ on steam-bath, the organic portion was successively extracted with $\mathrm{CHCl}_{3}$ $(3 \times 50 \mathrm{~mL})$, neutralized with $\mathrm{NaHCO}_{3}$, washed with water and dried $\left(\mathrm{CaCl}_{2}\right)$, and concentrated at reduced pressure to give the lactone $\mathbf{9}$ (3.43 g, $15.6 \mathrm{mmol}, 99.8 \%)$.

3.4.3. 4,4-Bis(3,4-dimethoxyphenyl)-2,3-dimethyl-butyric acid (10)

A solution of $\mathrm{AlCl}_{3}(2.23 \mathrm{~g}, 16.8 \mathrm{mmol})$ in veratrol $(1 \mathrm{~mL}$, $7.97 \mathrm{mmol}$ ) was slowly dropwise to veratrol $(1 \mathrm{~mL}, 7.97 \mathrm{mmol}$, $15 \mathrm{~min})$ and the lactone $\mathbf{9}(2.2 \mathrm{~g}, 8.78 \mathrm{mmol})$. The resulting mixture was then stirred at $80^{\circ} \mathrm{C}$ for $3 \mathrm{~h}$, and then the complex was decomposed by dropwise addition of EtOH $(5 \mathrm{~mL})$ at room temperature, followed by addition of $10 \% \mathrm{HCl}(10 \mathrm{~mL})$. After disappearance of solid particles, the mixture was extracted with $\mathrm{CHCl}_{3}(3 \times 50 \mathrm{~mL})$, washed with $8 \% \mathrm{NaHCO}_{3}(3 \times 50 \mathrm{~mL})$ and then with $10 \% \mathrm{HCl}$ until a white solution was obtained. The removal of solvents under reduced pressure yielded the intermediary acid $\mathbf{1 0}$ (3.20 g, $8.24 \mathrm{mmol}, 93.8 \%$ ).

\subsection{4. $8^{\prime}$-epi-Aristoligone and aristoligone $(\mathbf{4}+\mathbf{1 1})$}

The acid $10(3.02 \mathrm{~g}, 8.1 \mathrm{mmol})$ was dissolved in dry benzene $(6 \mathrm{~mL})$, then $\mathrm{PCl}_{5}(1.98 \mathrm{~g}, 9.5 \mathrm{mmol})$ was added to this solution, which was kept stirring in ice-bath for $30 \mathrm{~min}$. Then, the stirring mixture was warmed up to $40^{\circ} \mathrm{C}$ and occasionally shacked until the reactants dissolved. After that, the mixture was chilled and a solution of $\mathrm{SnCl}_{4}(2.84 \mathrm{~g}, 10.9 \mathrm{mmol})$ in dry benzene $(4 \mathrm{~mL})$ was added to it. In a few minutes, a red precipitate was observed, which was dissolved with concentrate $\mathrm{HCl}$. A mixture comprising the enantiomer pairs $\mathbf{4 + 1 1}(2.85 \mathrm{~g}, 7.7 \mathrm{mmol}, 95.1 \%)$ was obtained by extraction with $\mathrm{CHCl}_{3}$, followed by removal of the solvents.

\subsubsection{Aryltetralol 12}

To a stirring solution of $\mathbf{4 + 1 1}(2.00 \mathrm{~g}, 5.40 \mathrm{mmol})$ in $\mathrm{MeOH}$ $(20 \mathrm{~mL})$ a methanol solution of $\mathrm{NaBH}_{4}(204.2 \mathrm{mg}, 5.40 \mathrm{mmol}$, $20 \mathrm{~mL}$ ) was added. The mixture was stirred in an ice-bath for $4 \mathrm{~h}$. Following reduction, the excess of $\mathrm{NaBH}_{4}$ was quenched by dropwise addition of $\mathrm{MeOH}$ and water. The organic phase resulting from EtOAc $(3 \times 30 \mathrm{~mL})$ extraction was washed with water, dried $\left(\mathrm{MgSO}_{4}\right)$, and concentrated to yield $12(2.01 \mathrm{~g}, 5.40 \mathrm{mmol}$, $100.0 \%)$.

3.4.6. (-)-( $\left.7^{\prime} R, 8^{\prime} S\right)$ - and (+)-( $\left.7^{\prime} S, 8^{\prime} R\right)-3^{\prime}, 4^{\prime}, 4,5$-Tetramethoxy-2, $7^{\prime}-$ cyclolignan-7-ene [(-)-cyclogalgravin, 13a and (+)-cyclogalgravin, 13b]

To a stirring solution of the alcohol $12(1.93 \mathrm{~g}, 5.20 \mathrm{mmol})$ in dry benzene $(15 \mathrm{~mL}) \mathrm{TsOH}(0.72 \mathrm{~g}, 0.40 \mathrm{mmol})$ was slowly added. After stirring under reflux, this solution was cooled, extracted with $\mathrm{CHCl}_{3}$ and the solvent removed under reduced pressure to give $13(1.53 \mathrm{~g}, 4.33 \mathrm{mmol}, 83.3 \%)$. This product was subjected to chiral-HPLC analysis [cellulose tris-(3,5-dichlorophenylcarbamate column) to give $(-)-13 a(0.78 \mathrm{~g}, 2.20 \mathrm{mmol}$, ee $>99.9 \%)$, and (+)-13b (0.75 g, $2.13 \mathrm{mmol}$, ee > 99.9\%).

(-)-13a: yellow oil $\left(\mathrm{CHCl}_{3}\right) ;[\alpha]_{\mathrm{D}}^{25}-96$ (c 0.21, MeOH); (+)-13b: Yellow oil $\left(\mathrm{CHCl}_{3}\right) ;[\alpha]_{\mathrm{D}}^{25}+106$ (c $\left.0.20, \mathrm{MeOH}\right)$, lit. (Messiano et al., $2010)][\alpha]_{\mathrm{D}}^{25}-106$ (c 1.07, $\left.\mathrm{CHCl}_{3}\right) ; \mathrm{CD}\left(\mathrm{CHCl}_{3}, \mathrm{c} 0.3\right):[\theta]_{296}+24783$, $[\theta]_{290}+10406,[\theta]_{274}+71184,[\theta]_{249}-15366,[\theta]_{240}+543,[\theta]_{226}$ +71706, $[\theta]_{220}-52760,[\theta]_{210}-153856,[\theta]_{201}+113946$. 


\subsubsection{HPLC separation of $\mathbf{4 + 1 1}$}

Aliquots $(30 \mathrm{mg}$ ) of $\mathbf{4}+\mathbf{1 1}$ were subjected to semi-preparative HPLC (20 times) using RP-C18 ODS Chrompack column, UV $(\lambda 254 \mathrm{~nm})$ detector, flow rate of $8 \mathrm{~mL} / \mathrm{min}$, and $\mathrm{MeOH} / \mathrm{H}_{2} \mathrm{O}$ (7:3, $\mathrm{v} / \mathrm{v})$ as mobile phases.

\subsubsection{Chiral-HPLC analysis of $\mathbf{4}(\mathbf{4 a}+\mathbf{4 b})$ and $\mathbf{1 3}(\mathbf{1 3 a}+\mathbf{1 3 b})$}

Aryltetralone $(4,4 \mathrm{mg})$ and aryltetralene $(13,4 \mathrm{mg})$ lignans were subjected to HPLC (44 times each sample) using Chiralpak ${ }^{\circledR}$ IC column, $U V(\lambda 270 \mathrm{~nm})$ and polarimeter $(\lambda 365 \mathrm{~nm})$ detectors, flow rate of $0.5 \mathrm{~mL} / \mathrm{min}$ and $0.7 \mathrm{~mL} / \mathrm{min}$ for 4 and $\mathbf{1 3}$, respectively, and $n$-hexanes/EtOAc (17:3, v/v for 4 and 87:13, v/v for 13) as mobile phases (Fig. S5).

\section{Conclusions}

Two new lignans were isolated from $H$. reniformis. Aryltetralone, aryltetralol, and aryltetralene lignans were synthesized by a regioselective route in four, five, and six steps, successively, with good overall yields (81.0\%, 80.9\%, and 67.6\%). (-)-Cyclogalgravin (13a) and (-)-aristoligol (12a) exhibited activity ( IC $_{50} \sim 10.8$ and $8.4 \mu \mathrm{M}$, respectively), the latter exhibited lower toxicity.

\section{Acknowledgments}

The authors thank Vinicius C. Souza and Lindolpho Capellari Jr. for plant identification, and the Fundação de Amparo à Pesquisa do Estado de São Paulo (FAPESP), Coordenação de Aperfeiçoamento de Pessoal de Nível Superior (CAPES), and Conselho Nacional de Desenvolvimento Científico e Tecnológico (CNPq/MCT/MS/PRONEX, Brazil) for financial support and fellowships.

\section{Appendix A. Supplementary data}

Supplementary data associated with this article can be found, in the online version, at http://dx.doi.org/10.1016/j. phytol.2015.06.001.

\section{References}

Advanced Chemistry Development, 2010. ACD/C + H NMR predictors and DB: ChemSketch Window. Version 12.01. ACD, Toronto 1CD-ROM.

Barba, I., Chinchilla, R., Gomez, C., 1990. Electrochemical methoxylation of allyl and propenyl derivatives of phenol and phenol ethers. J. Org. Chem. 55, 3270-3272.

Blears, J.G., Haworth, R.D., 1958. The constituents of natural phenolic resins. 23. A synthesis of galgravin. J. Chem. Soc. 1985-1987.

Chavan, S.P., 2004. Reformatsky reaction of $\alpha$-chloroesters with carbonyl compounds with commercially available zinc. J. Chem. Res. Synop. 6, 406-407.

Crossley, N.S., Djerassi, C., 1962. Naturally occurring oxygen heterocyclics. 11. Veraguensin. J. Chem. Soc. 1459-1462. da Silva, T., Krettli, A.U., de Andrade-Neto, V.F., Lopes, L.M.X., 2004. Set of lignans and lignan extracts comprises constituents of malaria prevention compositions. BR200404986-A. Patent PI0404986-1, 2004. Lignanas, lignanas ariltetralônicas, extratos, processos de obtenção de lignanas, processo de obtenção de extratos, uso de lignanas, uso de extratos e composição farmacêutica para prevenir e tratar malária. Rev. Prop. Ind. 1774.

da Silva, T., Lopes, L.M.X., 2004. Aryltetralone lignans and 7,8-seco-lignans from Holostylis reniformis. Phytochemistry 65, 751-759.

da Silva, T., Lopes, L.M.X., 2006. Aryltetralol and aryltetralone lignans from Holostylis reniformis. Phytochemistry 67, 929-937.

de Andrade-Neto, V.F., da Silva, T., Xavier Lopes, L.M., do Rosario, V.E., de Pilla Varotti, F., Krettli, A.U., 2007. Antiplasmodial activity of aryltetralone lignans fro Holostylis reniformis. Antimicrob. Agents Chemother. 51, 2346-2350.

Iguchi, M., Nishiyama, A., Hara, M., Terada, Y., Yamamura, S., 1978. Anodic-oxidation of E-isoeugenol and Z-isoeugenol. Chem. Lett. 1015-1018.

Krettli, A.U., Adebayo, J.O., Krettli, L.G., 2009. Testing of natural products and synthetic molecules aiming at new antimalarials. Curr. Drug Targets 10, 261-270.

Konishi, T., Konoshima, T., Daikonya, A., Kitanaka, S., 2005. Neolignans from Piper futokadsura and their inhibition of nitric oxide production. Chem. Pharm. Bull. 53, 121-124.

Kuo, Y.H., Lin, S.T., 1993. Studies on chromium trioxide-based oxidative coupling reagents and synthesis of lignan-cagayanone. Chem. Pharm. Bull. 41,1507-1512.

Kuo, Y.H., Lin, S.T., Wu, R.E., 1989. 3 New lignans from the nutmeg of Myristica cagayanesis. Chem. Pharm. Bull. 37, 2310-2312.

Lopes, L.M.X., Pereira, M.D.P., da Silva, T., Krettli, A.U., 2012. Sesquiterpenes from Holostylis reniformis. Pharm. Microbiol. 50, 643-644.

Martins, G.F., Pereira, M.D.P., Lopes, L.M.X., da Silva, T., Vieira e Rosa, P. de T., Barbosa, F.P., Messiano, G.B., Krettli, A.U., 2014. Intraspecific variability of Holostylis reniformis: concentration of lignans, as determined by maceration and supercritical fluid extraction $\left(\mathrm{SFE}-\mathrm{CO}_{2}\right)$, as a function of plant provenance and plant parts. Quim. Nova 37, 281-287.

Messiano, G.B., da Silva, T., Nascimento, I.R., Lopes, L.M.X., 2008. Biosynthesis of antimalarial aryltetralone lignans fro Holostylis reniformis. Planta Med. 74, 924-924.

Messiano, G.B., da Silva, T., Nascimento, I.R., Lopes, L.M.X., 2009. Biosynthesis of antimalarial lignans from Holostylis reniformis. Phytochemistry 70, 590-596.

Messiano, G.B., Wijeratne, E.M.K., Lopes, L.M.X., Gunatilaka, A.A.L., 2010. Microbial transformations of aryltetralone and aryltetralin lignans by Cunninghamella echinulata and Beauveria bassiana. J. Nat. Prod. 73, 1933-1937.

Müller, A., Vajda, M., 1952. Dimeric propenyl phenol ethers. 15. The synthesis of 1-veratryl-2,3-dimethyl-6,7-dimethoxytetralin. J. Org. Chem. 17, 800-806.

Noedl, H., Wernsdorfer, W.H., Miller, R.S., Wongsrichanalai, C., 2002. Histidine rich protein II, a novel approach to antimalarial drug susceptibility testing. Antimicrob. Agents Chemother. 46, 1658-1664.

Peng, Y., Luo, Z.B., Zhang, J.J., Luo, L., Wang, Y.W., 2013. Collective synthesis of several $2,7^{\prime}$-cyclolignans and their correlation by chemical transformations. Org. Biomol. Chem. 11, 7574-7586.

Pereira, M.D.P., da Silva, T., Lopes, L.M.X., Krettli, A.U., Madureira, L.S., ZukermanSchpector, J., 2012. 4,5-Seco-guaiane and a nine-membered sesquiterpene lactone from Holostylis reniformis. Molecules 17 (14), 046-14057.

Purushothaman, K.K., Sarada, A., Connolly, J.D., 1984. Structure of malabaricanol-a lignan from the aril of Myristica malabarica Lam. Indian J. Chem. Sect. B 23, $46-48$.

Rye, C.E., Barker, D., 2011. Asymmetric synthesis of (+)-galbelgin, (-)-kadangustin J, (-)-cyclogalgravin and (-)-pycnanthulignenes A and B, three structurally distinct lignan classes, using a common chiral precursor. J. Org. Chem. 76, 6636-6648.

Takeya, T., Matsumoto, H., Kotani, E., Tobinaga, S., 1983. New reagent systems containing $\mathrm{CrO}_{3}$ provide precursors for syntheses of neo-lignans. Chem. Pharm. Bull. 31, 4364-4367.

Yvon, B.L., Datta, P.K., Le, T.N., Charlton, J.L., 2001. Synthesis of magnoshinin and cyclogalgravin: modified Stobbe condensation reaction. Synthesis 1556-1560. 